\title{
Formation shape transition of multiple mobile robots in leader- follower method
}

\author{
Atsushi Fujimori ${ }^{1}$, Kotaro Oh-kiri ${ }^{2}$, Shinsuke Oh-hara ${ }^{3}$ \\ ${ }^{1,3}$ Department of Mechanical Engineering, University of Yamanashi, Japan \\ ${ }^{2}$ Terumo Corporation, Japan
}

\begin{abstract}
Article Info
Article history:

Received May 3, 2021

Revised Jul 15, 2021

Accepted Jul 23, 2021

Keywords:

Formation shape transition

Leader-follower method

Multiple mobile robots

Sonar sensor

ABSTRACT

This paper presents a formation shape transition technique of multiple mobile robots in the leader-follower method as a new function that gives flexibility to the formation control of mobile robots with multiple sonars. First, we propose basic shape transition methods for the case of two mobile robots under formation control by the leader-follower method, and then extend the methods to the shape transition of three mobile robots. Since the multiple sonars attached to the mobile robot are located forward, including the left and right sides, there is a constraint on the formation shape feasible by the leaderfollower method. In the case of two mobile robots, the follower must be positioned behind the leader. Therefore, there are three shapes of the follower relative to the leader: line, right-back, left-back. In the case of three mobile robots, three types of line, zigzag, triangle shapes are considered. The effectiveness of the proposed technique is demonstrated by experiments using real mobile robots.
\end{abstract}

This is an open access article under the CC BY-SA license.

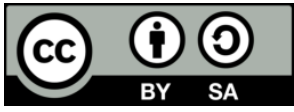

Corresponding Author:

Atsushi Fujimori

Department of Mechanical Engineering

University of Yamanashi

4-3-11 Takeda, Kofu, Yamanashi, 400-8511, Japan

Email: afujimori@yamanashi.ac.jp

\section{INTRODUCTION}

Formation Control of multiple mobile robots has attracted a great deal of attention in recent years. This is due to the fact that formation guidance is one way of efficient transportation systems such as automobiles and airplanes, and that it can become a system that brings about more efficient work achievement than a single robot [1]-[4]. As control approaches to realize formation control, behavior-based methods [5]-[7], multi-agent systems [8]-[11], virtual structure methods [12], [13], leader-follower methods [14]-[18], etc. are generally known. This paper is a study on the formation control of multiple mobile robots using the leader-follower method. In the leader-follower method, multiple mobile robots are classified into a leader and followers. The control purpose of the leader is to make configuration and route selection of the formation, while the control purpose of the followers is to control so that the relative positional relationship with the leader is kept at a specified reference. Therefore, it can be said that the subject examined in the leader-follower method is to design control systems on the followers.

The authors have been studying various subjects in the formation control by the leader-follower method using mobile robots with multiple sonar sensors which are described as follows [19]-[24]. Generally, since the formation control law based on the dynamic inversion [25] included the states of both leader and follower [14]-[16], the follower needed means for obtaining the state of the leader to implement the control law on real mobile robots. As one way to avoid this, we proposed self-made input (SMI), which was an 
autonomous decentralized control law utilizing an estimate of the state of the leader [19], [20]. The SMI did not need any communication means between the mobile robots and improved the applicability of the leaderfollower method. We also studied formation control in obstacle scattered environments [21]-[24]. In these studies, multiple sonars were adaptively used for two purposes: leader tracking for formation control and obstacle detection to avoid collision with obstacles. The formation control was then realized while avoiding obstacles even in the presence of obstacles [21], [22]. Furthermore, we developed a formation control method for large obstacle avoidance in which modules reinforced for the avoidance were introduced [23], [24].

One of the new problems with the formation control of mobile robots is to develop a function that can change the formation shape as necessary. In this paper, we call this function "shape transition". In the formation control with large obstacle avoidance described above, an algorithm was proposed in which the local part of the shape was temporarily changed to a shape that easily avoided obstacles and then returned to the original shape [23], [24]. It may also be desirable from the practical point of view to change the formation shape to reconstruct a formation by adding or removing mobile robots forming the formation. It can be said that the shape transition is a function that improves the flexibility of the formation control of the mobile robots. For these reasons, this paper investigates the shape transition method of multiple mobile robots during formation control by the leader-follower method. First, we propose basic shape transition methods under formation control for the case of two mobile robots. These methods are extended to the shape transition of three mobile robots. The effectiveness of the proposed technique is verified by experiments using a real mobile robot Pioneer with multiple sonar sensors.

\section{FORMATION CONTROL OF MOBILE ROBOT WITH SONAR SENSOR}

\subsection{Leader-follower method}

Consider a mobile robot with the non-holonomic constraint. The position of the robot moving in the two-dimensional plane is represented by a stationary Cartesian coordinate system $(x(t), y(t))$, where $t$ is continuous time. The direction angle of the robot is $\theta(t)(-\pi \leq \theta(t)<\pi$ [rad] $)$, which is measured from the positive $x$-axis. The linear and angular velocities are $v(t)$ and $\omega(t)$, respectively, which are the control inputs of the mobile robot. The equation of kinematics for the mobile robot is given by (1).

$$
\left\{\begin{array}{c}
\dot{x}=v(t) \cos \theta(t) \\
\dot{y}=v(t) \sin \theta(t) \\
\dot{\theta}=\omega(t)
\end{array}\right.
$$

Where the magnitude of the control input is constrained by (2).

$$
|v(t)| \leq v_{\max } \quad \max
$$

$v_{\max }$ and $\omega_{\max }$ are the maximums of $v(t)$ and $\omega(t)$, respectively. Figure 1 shows how two mobile robots are controlled by the leader-follower method, where $L$ and $F$ are the leader and follower robots, respectively. Hereafter, subscripts ' $l$ ' and ' $f$ attached to variables refer to the leader and the follower, respectively. Both are governed by the kinematical equations that rewrite the variables in (1) into their variables, respectively. The relative distance between the leader and follower is denoted as $d_{l}\left(=d_{f}\right)$ and the relative angles from their headings are denoted as $\gamma_{l}$ and $\gamma_{f}$, respectively. The purpose of the formation control by the leader-follower method is to navigate multiple mobile robots from start to goal while forming a formation shape. The follower is then controlled to keep $d_{f}$ and $\gamma_{f}$ at their references $d_{f}^{\text {ref }}$ and $\gamma_{f}^{\text {ref }}$, respectively. Fujimori et al. proposed SMI, which was an autonomous decentralized control law utilizing the estimated state of the leader [19], [20]. The SMI did not require any communication means between the leader and follower. We also use the SMI for the formation control law in this paper.

\subsection{Mobile robot pioneer}

Figure 2 shows photographs of mobile robots Pioneer-1 and Pioneer-3 used in formation control in this paper [26]-[28]. Pioneer-1 shown in the photographs on the left and middle are 450 [mm] in length, 360 $[\mathrm{mm}]$ in width for the left and right wheels, and 225 [mm] in height. Pioneer-3 shown in the photograph on the right is slightly larger than Pioneer-1. The drive system of Pioneer- 1 and Pioneer- 3 is provided by two independent, reversely rotatable DC motors on the left and right wheels. Pioneer-1 has seven ultrasonic sonars, while Pioneer-3 has eight ultrasonic sonars. Table 1 shows the attached angle of the sonars on Pioneer-1 and Pioneer-3. 


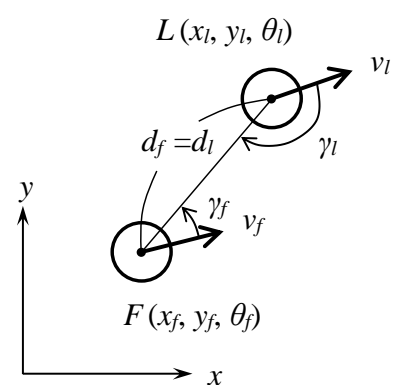

Figure 1. The relative position between the leader and follower robots
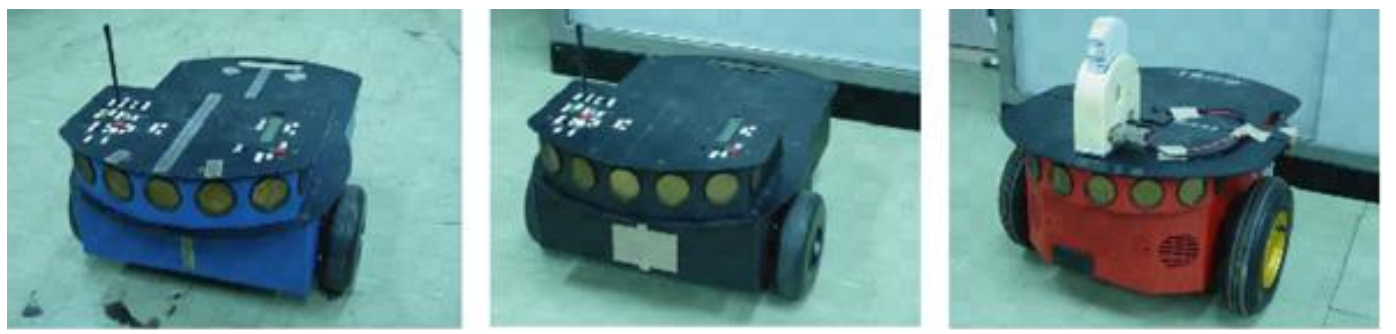

Figure 2. Pioneer-1 and -3

Table 1. Attached are angles of sonars in Pioneer-1 and -3

\begin{tabular}{ccc}
\hline \multirow{2}{*}{ No. of sonar } & \multicolumn{2}{c}{$\varphi_{i}[\mathrm{deg}]$} \\
& Pioneer-1 & Pineer-3 \\
\hline 0 & 90 & 90 \\
1 & 30 & 50 \\
2 & 15 & 30 \\
3 & 0 & 10 \\
4 & -15 & -10 \\
5 & -30 & -30 \\
6 & -90 & -50 \\
7 & - & -90 \\
\hline
\end{tabular}

In the formation control with obstacle avoidance [22]-[24], the multiple sonars were used not only to detect obstacles for collision avoidance but also to obtain the relative distance and angle with the leader robot for formation control. This function was called "adaptive sonar assignment" [22]-[24]. Since this paper does not consider obstacle avoidance, the sonars are used for only obtaining the relative distance and angle. Assuming that $s_{i}$ is the output value of the $i$-th sonar and $\phi_{i}$ is the attached angle of the $i$-th sonar, the relative distance $d_{f}$ and angle $\gamma_{f}$ are obtained by averaging the weighted output of the fired sonars. That is (3) and (4).

$$
\begin{gathered}
d_{f}=\frac{\sum_{i \in \mathscr{F}} w_{i} s_{i}}{\sum_{i \in \mathscr{F}} w_{i}} \\
\gamma_{f}=\frac{\sum_{i \in \mathscr{F}} w_{i} \phi_{i}}{\sum_{i \in \mathscr{F}} w_{i}}
\end{gathered}
$$

Where $w_{i}$ is the weighting coefficient and $\mathcal{F}$ means the set of sonar numbers for leader track. See [22] for details.

In the previous studies dealing with obstacle avoidance [22]-[24], the follower estimated the position of the leader during formation control, called "leader estimate", and used to set the target position for reconstructing the formation after avoiding the obstacle and to re-detect the leader. "Leader estimate" will be included in the proposed control algorithm because the same procedure will be needed in the shape transition described in the next section. 


\section{FORMATION SHAPE TRANSITION IN LEADER-FOLLOWER METHOD}

In this section, we first propose basic shape transition methods for the case of two mobile robots under formation control by the leader-follower method. The extension to the case of three mobile robots is next shown. As mentioned in Section 2.2, the sonars attached to the mobile robot are located forward, including the left and right sides, and have the non-holonomic constraint in the kinematics, so that there is a constraint on the formation shape feasible by the leader-follower method. In the case of two mobile robots, the follower must be positioned behind the leader. Therefore, there are three shapes of the follower relative to the leader: line, right-back, left-back. In the case of three mobile robots, three types of line, zigzag, triangle shapes are considered. When the number of mobile robots increases further, there may be a variety of shapes as a whole, but locally it is possible to regard a combination of the shapes in the cases of two or three mobile robots. Therefore, the formation shape transition considered in this paper can be applied to the transition in general shape. In the formation control considered in this paper, it is supposed that the leader is moving at a constant speed in a certain direction.

\subsection{Shape transition in two mobile robots}

This paper proposes two shape transition methods described below according to transition movement, which refers to the amount of shape transition and is quantitatively defined as the integral value of the control input. It is also defined as the time required to achieve the shape transition. However, their exact definitions are not necessarily useful, so we will discuss the shape transition only from a qualitative point of view. First, we propose two shape transition methods and then mention their application conditions.

\subsubsection{Shape transition for small transition movement (M-1)}

Figure 3 shows the shape transition from line to right-back as an example that the transition movement is small. $L$ and $F$ represent the leader and follower, respectively, and illustrate the transition behavior for each step. The black block arrow means the shift to the next step. At the beginning of the shape transition shown in Step-1, $F$ changes the referenced relative distance and angle for the line to the distance and angle for the right-back. It is denoted as "New $d_{f}^{r e f}, \gamma_{f}^{\text {ref }}$ " in Figure 3. As the result, the transition to fight back is achieved by shifting to the right while continuing the formation control. This behavior is denoted as "on FC".

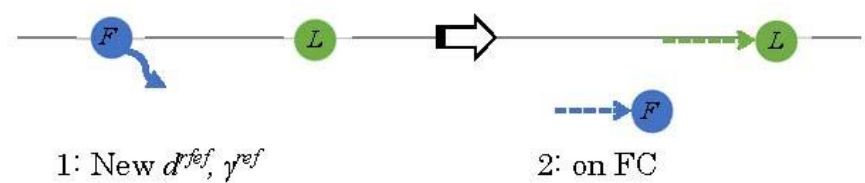

Figure 3. Shape transition from line to right-back by M-1

\subsubsection{Shape transition for large transition movement (M-2)}

Figure 4 shows the shape transition from right-back to left-back when the transition movement is large. In this case, the shape transition is surely performed by interrupting the formation control. The position where the follower should move is first defined. This is called target position (TP). The direction angle of $F$ and the estimated position of $L$ obtained by "leader estimate" [22]-[24] are used for defining the TP. The transition to the TP is then performed. The specific process is shown below in the discrete-time representation with constant sampling time, denoted as $T_{s}$; that is, $t_{k}$ represents the $k$-th sampled discrete-time.

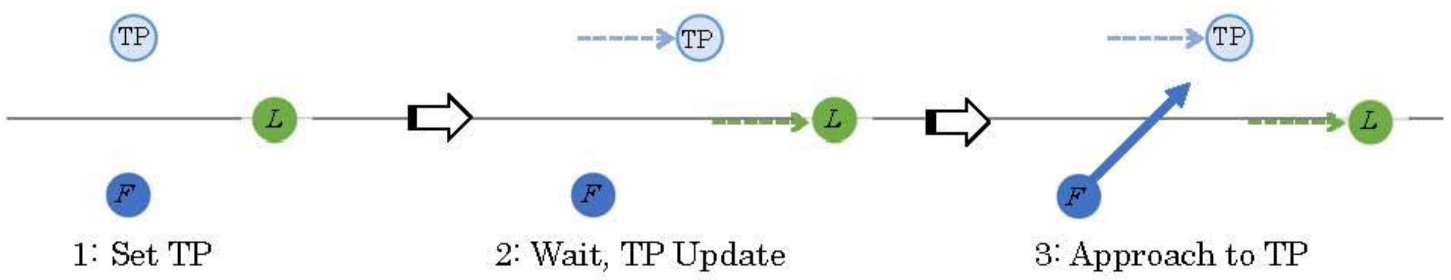

Figure 4. Shape transition from right-back to left-back by M-2 
Step-1: Suppose the starting time of the shape transition is $t_{k}=t_{k 1}$. Letting the referenced relative distance and angle for left-back be $d_{f}^{r e f}$ and $\gamma_{f}^{r e f}$, and the estimated position of $L$ be $\left(\hat{x}_{l}\left(t_{k 1}\right), \hat{y}_{l}\left(t_{k 1}\right)\right)$, the TP is given by (5).

$$
\left\{\begin{array}{l}
x_{p}\left(t_{k 1}\right)=\hat{x}_{l}\left(t_{k 1}\right)-d_{f}^{r e f} \cos \left(\theta_{f}\left(t_{k 1}\right)+\gamma_{f}^{r e f}\right) \\
y_{p}\left(t_{k 1}\right)=\hat{y}_{l}\left(t_{k 1}\right)-d_{f}^{r e f} \sin \left(\theta_{f}\left(t_{k 1}\right)+\gamma_{f}^{r e f}\right)
\end{array}\right.
$$

It is denoted as "set TP" in Figure 4.

Step-2: The movement of $F$ pauses for a specified while. It is denoted as "Wait". For $t_{k} \geq t_{k 1}$, the TP is updated by (6).

$$
\left\{\begin{array}{l}
x_{p}\left(t_{k}\right)=x_{p}\left(t_{k-1}\right)+v_{0} T_{s} \cos \theta_{f}\left(t_{k 1}\right) \\
y_{p}\left(t_{k}\right)=y_{p}\left(t_{k-1}\right)+v_{0} T_{s} \sin \theta_{f}\left(t_{k 1}\right)
\end{array}\right.
$$

It is denoted as "TP update" in Figure 4.

Step-3: Suppose that "Wait" is finished at $t_{k}=t_{k 2}\left(>t_{k 1}\right)$. After that $\left(t_{k}>t_{k 2}\right), F$ moves to the TP according to the following control law,

$$
\begin{aligned}
& v_{f}\left(t_{k}\right)=v_{\max } \\
& \omega_{f}\left(t_{k}\right)=k_{T}\left(\theta_{p}\left(t_{k}\right)-\theta_{f}\left(t_{k}\right)\right) \\
& \theta_{p}\left(t_{k}\right) \triangleq \tan ^{-1} \frac{y_{p}\left(t_{k}\right)-y_{f}\left(t_{k}\right)}{x_{p}\left(t_{k}\right)-x_{f}\left(t_{k}\right)}
\end{aligned}
$$

where $k_{T}$ is a positive constant gain. This behavior is denoted as "Approach to TP". Step-4: After arriving at the TP, $F$ returns to formation control by re-detecting $L$.

\subsubsection{Selection of shape transition method}

M-1 only changes the referenced relative distance and angle for the new shape and performs the shape transition while continuing the formation control, denoted as "on FC" in Figure 3. For applying M-1, it is necessary to satisfy the following conditions that i) the leader to follow is within the range of sonars detectable relative to the follower and ii) the referenced relative distance and angle of the new shape are within the fluctuation range which can correspond with the control law of the leader-follower method; that is, within the range which does not cause input saturation. Otherwise, M-2 is selected for shape transition. The reason why "Wait" is included in step-2 of M-2 is that it is difficult for a mobile robot with the nonholonomic constraint to move smoothly immediately after "Set TP". It is, therefore, a means to wait until the TP becomes a position that is easy to move. Also, "TP Update" given by (6) assumes that the leader is moving at a constant velocity $v_{0}$ without changing its direction.

Figure 5 shows the flow chart of the follower control algorithm for formation control and shape transition summarized above. Triggering shape change branches to the formation control or the shape transition at "Shape Change". The former is called N-mode, while the latter is called T-mode. N-mode contains modules for the formation control described in Section 2, which sequentially executes "adaptive sonar assignment", "leader estimate", "SMI" and generates the control inputs $v_{f}(t)$ and $\omega_{f}(t)$ to the follower. In T-mode, on the other hand, the shape transition method is selected by "transition method (TM) select". For M-2, $v_{f}(t)$ and $\omega_{f}(t)$ are generated from the process described in the previous section. The estimated position of the leader, which has been obtained at "leader estimate" in N-mode, is utilized at "Set TP" in M-2. There are two cases to select M-2 for the shape transition on two mobile robots: right-back $\rightarrow$ left-back or left-back $\rightarrow$ right back. Otherwise, $\mathrm{M}-1$ is selected. 


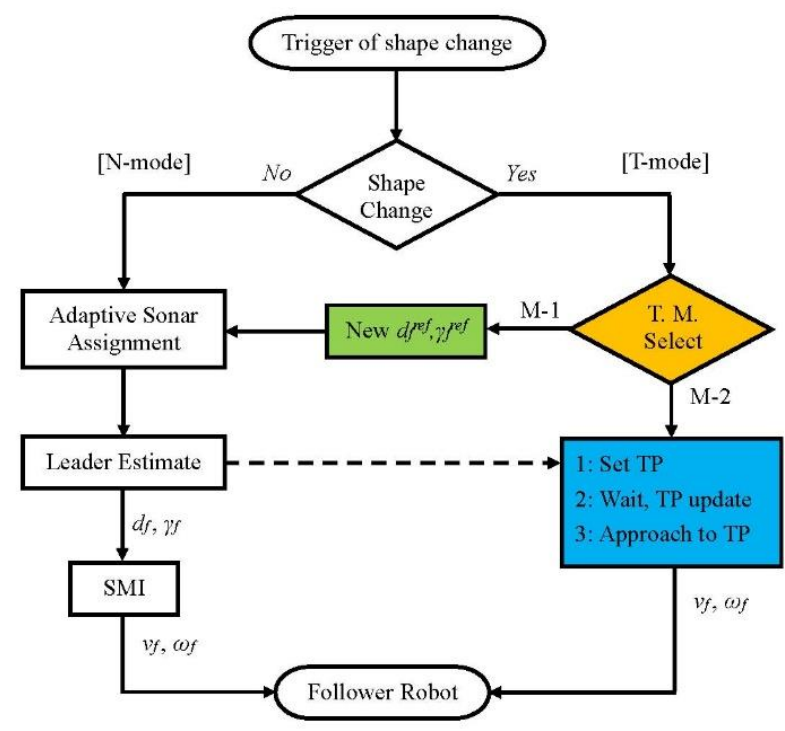

Figure 5. Follower control algorithm for formation control and shape transition

\subsection{Application to three mobile robots}

The proposed shape transition methods are applied to the cases of three mobile robots. In these cases, there are two follower robots which are referred to as $F_{1}$ and $F_{2}$ hereafter. It is assumed in this paper that the shape transition of $F_{1}$ is superior to that of $F_{2}$. The priority of the shape transition is not changed under formation control.

\subsubsection{Line $\rightarrow$ triangle}

Figure 6 shows the shape transition from line to the triangle. Since the transition movement of $F_{1}$ is small, M-1 is applied to $F_{1}$. The referenced relative distance and angle for the line are changed to the distance and angle for the triangle in step- 1 . On the other hand, since the transition movement of $F_{2}$ is large, M-2 is basically applied to $F_{2}$. Therefore, $F_{2}$ first sets TP for triangle allocation. If $F_{2}$ starts the transition behavior immediately, it is risky to collide with $F_{1}$. To avoid this, "wait" is inserted in step-2 and the position of the TP is updated during that time. $F_{2}$ moves to the TP when there is almost no risk of contacting $F_{1}$.

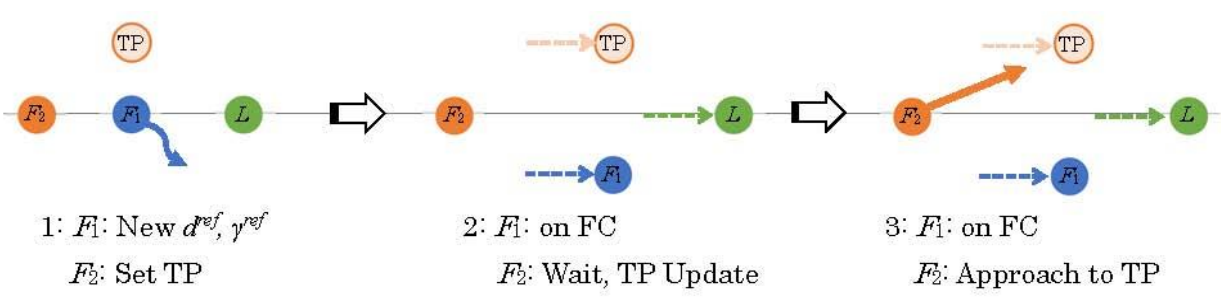

Figure 6. Shape transition from line to a triangle

\subsubsection{Triangle $\rightarrow$ line}

Figure 7 shows the shape transition from triangle to the line. M-1 is applied to both $F_{1}$ and $F_{2}$ because the transition movement is small. However, since $F_{2}$ needs to consider the non-holonomic constraint and risk-avoidance of collision with $F_{1}$, "wait" is inserted in Step-1. After "wait" is finished, the referenced relative distance and angle for the triangle are changed to the distance and angle for line, denoted as "New $d_{f}^{\text {ref }}, \gamma_{f}^{\text {ref }}$ " in step-2.

\subsubsection{Line $\rightarrow$ zigzag}

Figure 8 shows the shape transition from line to zigzag. Since the transition movement of $F_{1}$ is small, M-1 is applied to $F_{1}$. On the other hand, since the transition movement of $F_{2}$ is also small, the 
application of M-1 to $F_{2}$ is reasonable. However, the position of $F_{2}$ in zigzag after the transition is almost the same as that in line. Therefore, it is possible to reduce waste of movement by continuing the previous action for a while. This continuous action is called "self". The referenced relative distance and angle for the line are changed to the distance and angle for zigzag.

\subsubsection{Zigzag $\rightarrow$ line}

Figure 9 shows the shape transition from zigzag to the line. M-1 is applied to both $F_{1}$ and $F_{2}$ because the transition movement is small. Similar to the transition from line to zigzag shown in Figure 8, "Self" for $F_{2}$ is introduced to reduce unnecessary transition movement.

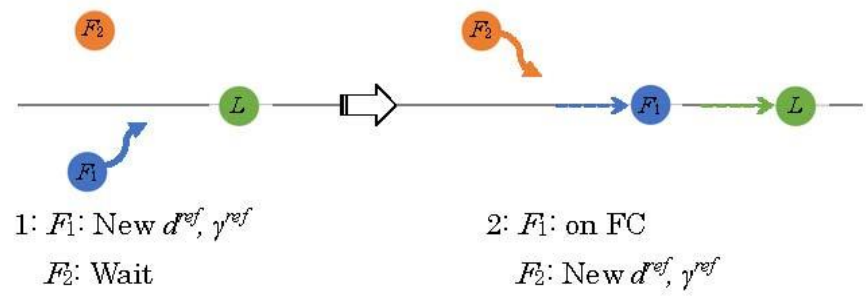

Figure 7. Shape transition from triangle to line

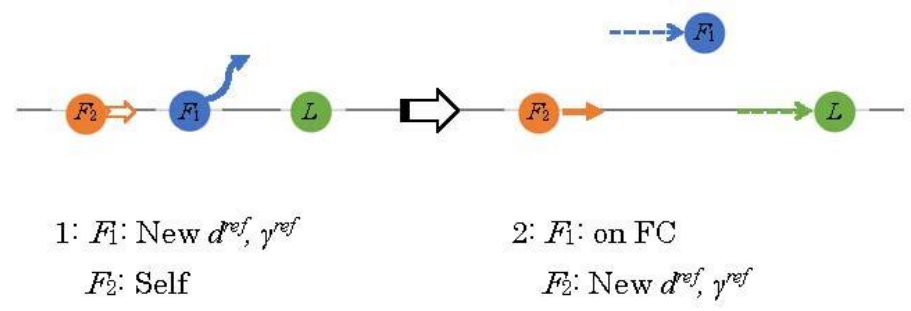

Figure 8. Shape transition from line to zigzag

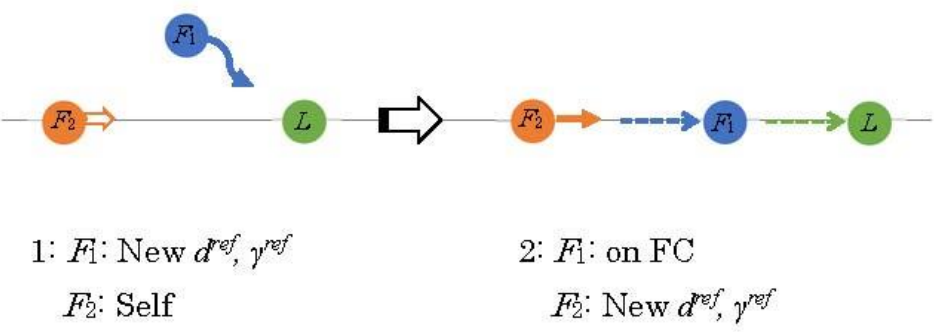

Figure 9. Shape transition from zigzag to line

\subsubsection{Triangle $\rightarrow$ zigzag}

Figure 10 shows the shape transition from triangle to zigzag. Performing the transition from triangle to zigzag directly, a collision may have occurred because the transition behavior of $F_{1}$ and $F_{2}$ is interrupted each other. The risk of colliding between the followers is then avoided by passing through-line on the way. The transition behavior shown in Figure 10 is therefore constructed by combining with that in Figure 7 and Figure 8.

\subsubsection{Zigzag $\rightarrow$ triangle}

Figure 11 shows the shape transition from zigzag to the triangle. Similar to the transition from triangle to zigzag, the risk of colliding between the followers is avoided by passing through-line on the way. The transition behavior shown in Figure 11 is therefore constructed by combining with that in Figure 6 and Figure 9.

Formation shape transition of multiple mobile robots in leader-follower method (Atsushi Fujimori) 

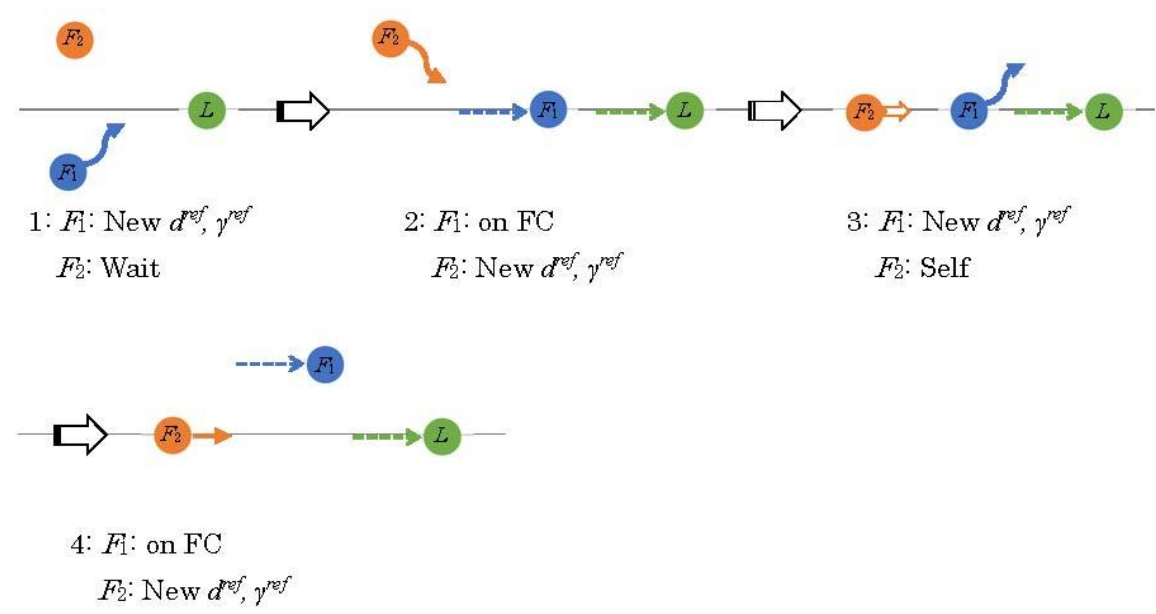

Figure 10. Shape transition from triangle to zigzag
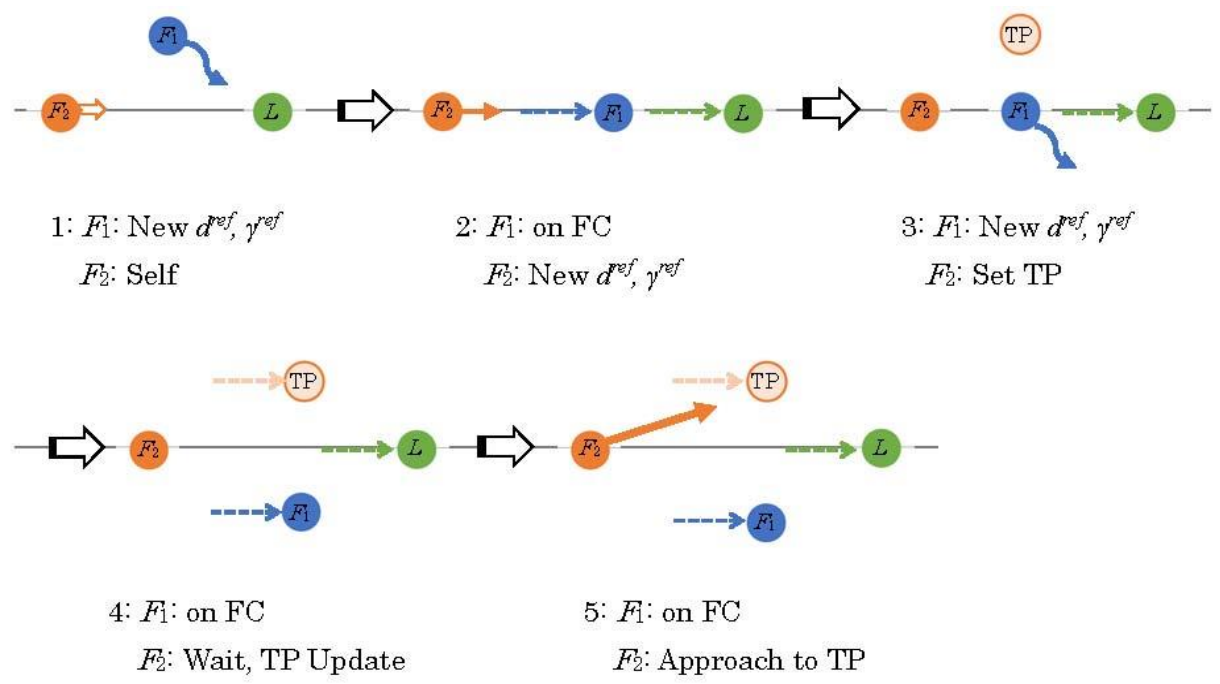

Figure 11. Shape transition from zigzag to triangle

\section{SHAPE TRANSITION EXPERIMENT}

To demonstrate the proposed shape transition methods, this section presents experimental results using three mobile robots, two pioneer-1 and one pioneer-3 which are shown in Figure 2. The blue pioneer-1 was used as a leader robot, denoted as $L$, the black Pioneer-1 as the first follower robot, denoted as $F_{1}$, and the red pioneer-3 as the second follower robot denoted as $F_{2}$. The referenced relative distance and angle for the formation shape; line, zigzag, and triangle, were given as follows.

* Line (Shape no.=71)

$$
\begin{array}{ll}
d_{f 1}{ }^{r e f}=600[\mathrm{~mm}] & \gamma_{f 1}{ }^{r e f}=0[\mathrm{deg}] \\
d_{f 2}{ }^{r e f}=600[\mathrm{~mm}] & \gamma_{f 2}{ }^{r e f}=0[\mathrm{deg}] \\
\text { (Shape no. }=72) & \\
d_{f 1}{ }^{r e f}=600[\mathrm{~mm}] & \gamma_{f 1}{ }^{r e f}=-20[\mathrm{deg}] \\
d_{f 2}{ }^{r e f}=600[\mathrm{~mm}] & \gamma_{f 2}{ }^{r e f}=20[\mathrm{deg}] \\
& \\
\hline l e(\text { Shape no. }=73) & \gamma_{f 1}{ }^{r e f}=15[\mathrm{deg}] \\
d_{f 1}{ }^{r e f}=600[\mathrm{~mm}] & \gamma_{f 2}{ }^{r e f}=-30[\mathrm{deg}] \\
d_{f 2}{ }^{r e f}=600[\mathrm{~mm}] &
\end{array}
$$

* Zigzag (Shape no.=72)

* Triangle (Shape no.=73) 
There are various reasons for the purpose of carrying out the shape transition such as obstacle avoidance, transportation efficiency, and addition or reduction of robots. Furthermore, it may be possible to consider the command generation of the shape transition from the leader or followers. Since this paper focuses on demonstrating the effectiveness of the proposed shape transition methods, in the experiments shown below, the triggering of the shape change is given by a simple method; that is, the travel distance (position) of the follower. In Section 3, the shape transition methods for the cases of two and three mobile robots were described. Since M-1 and M-2 proposed on the two mobile robots are embedded in the transition behavior of $F_{1}$ and $F_{2}$ for the cases of three mobile robots, the following in this section shows the experimental results of three mobile robots.

\subsection{Experiment 1: line $\rightarrow$ triangle}

Figures 12-17 show the experimental results of a shape transition from line to the triangle. The solid lines in Figure 12 represent the loci of movement of three mobile robots, respectively. The light-blue and magenta dashed lines indicate the estimated location of $L$ by $F_{1}$ and $F_{2}$. The marks; triangle, diamond, etc. indicate the positions to be noticed in the transition. The state at those times corresponds to the marks in Figures 13-17. The starting position of $L$ was the origin in the $(x, y)$ coordinate system, and the goal position was $(x, y)=(5000,-1500)[\mathrm{mm}]$. The upper figure in Figure 13 shows the time history of the mode flag selected by $F_{1}$ and $F_{2}$, which is listed in Table 2. The lower figure shows the time history of formation shape; that is, shape no.

Table 2. Mode flag

\begin{tabular}{cc}
\hline Mode flag & Mode \\
\hline 19 & "Self" in T-mode \\
20 & N-mode \\
$21-28$ & A-mode *) \\
29 & "Wait" in T-mode \\
30 & "Approach to TP" in T-mode \\
\hline
\end{tabular}

*) A-mode is not used in this paper.

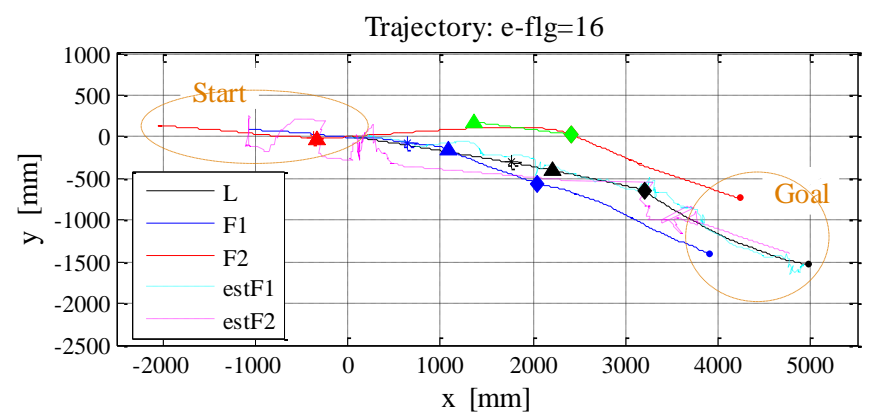

Figure 12. Traces of $L, F_{1}$, and $F_{2}$, and traces of leader position estimated by $F_{1}$ and $F_{2}$, line $\rightarrow$ triangle
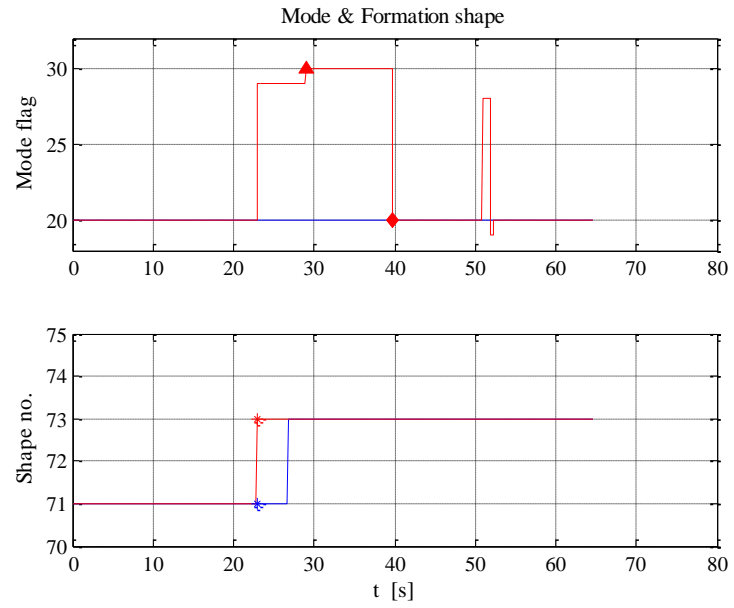

Figure 13. Time histories of mode flag and shape number, line $\rightarrow$ triangle 


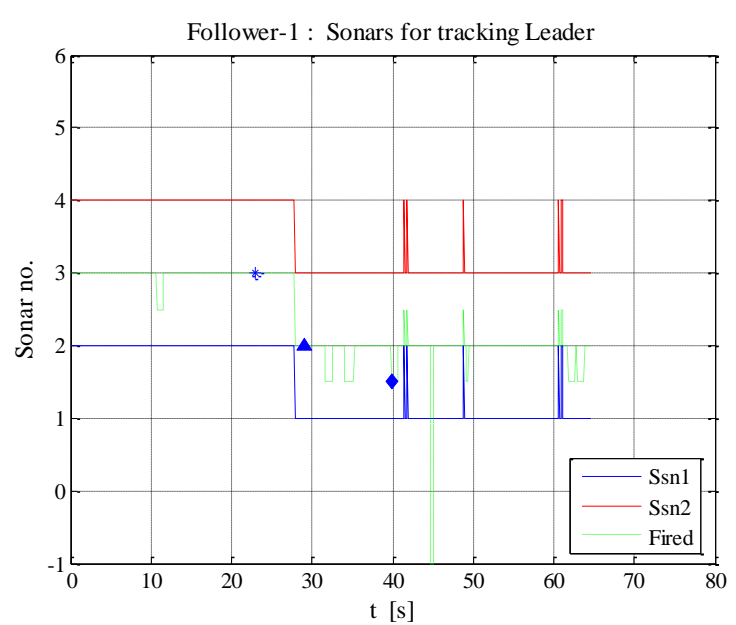

Figure 14. Time histories of sonar number for tracking leader, $F_{1}$, line $\rightarrow$ triangle

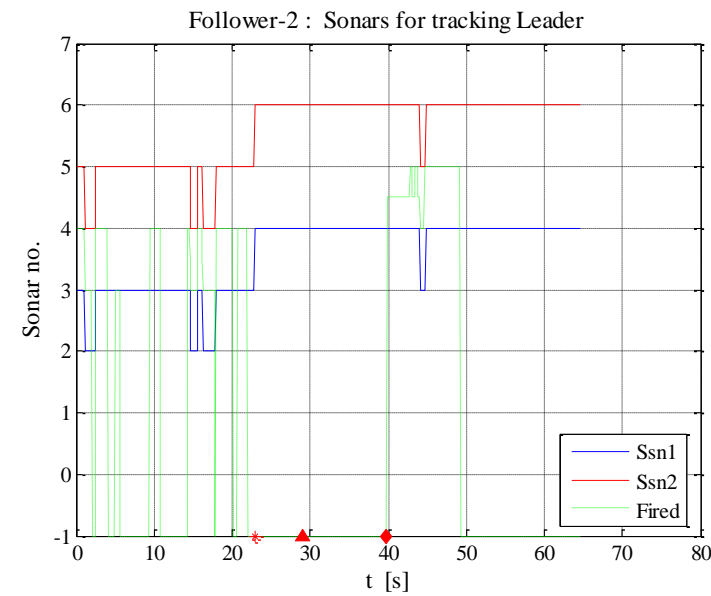

Figure 15 . Time histories of sonar number for tracking leader, $F_{2}$, line $\rightarrow$ triangle

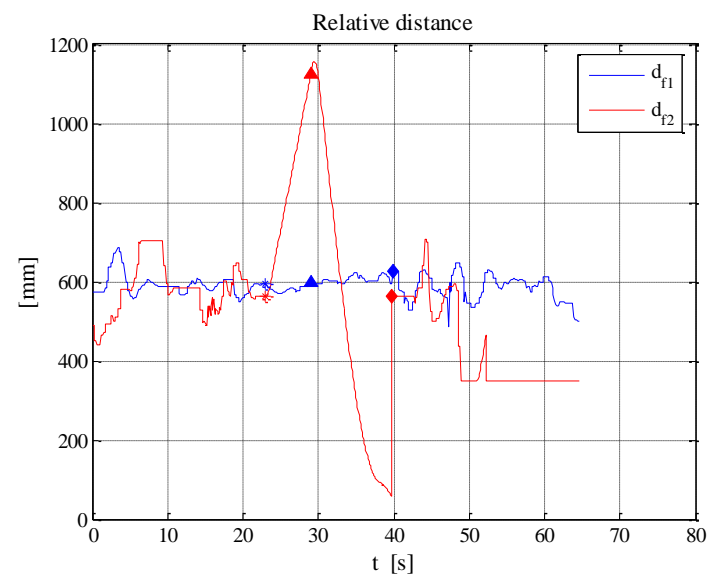

Figure 16. Time histories of relative distance $d_{f}$, line $\rightarrow$ triangle 


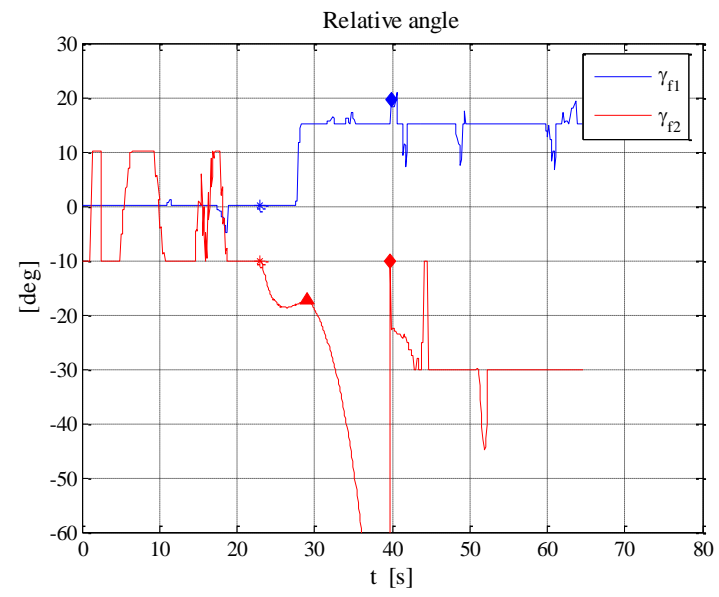

Figure 17. Time histories of relative angle $\gamma_{f}$ line $\rightarrow$ triangle

As shown in Figure 12, the three mobile robots started with forming Line. Then, as shown in Figure $13, F_{2}$ changed the shape from line to triangle at $t=23$ [s] (shape no.= $71 \rightarrow 73$ ). Then, the TP was set at the position marked by the green triangle, and "wait" (mode flag $=29$ ) was set. Mode flag was changed to 30 at $t=29[\mathrm{~s}]$; that is, "approach to TP" started. The position of the TP was updated as shown by the green solid line in Figure 12. $F_{2}$ returned to $\mathrm{N}$-mode at $t=40[\mathrm{~s}]$ (red diamond) and reached the goal in the form of a triangle. On the other hand, $F_{1}$ had always completed the shape transition in N-mode. Figure 14 and Figure 15 are the time histories of the lower limit (Ssn1) and upper limit (Ssn2) of the sonar number used to detect the leader. Also, when Fired (green dashed line) is between Ssn1 and Ssn2, it means that the leader is being detected by the sonars. Although $F_{2}$ was temporarily unable to detect $L$ (Fired =-1) during formation control, the formation control was stably performed because it took only a small amount of time. Figure 16 and Figure 17 show the time histories of the relative distance and angle. The relative distance $d_{f 1}$ and angle $\gamma_{f 1}$ of $F_{1}$ almost showed the same as their references. On the other hand, the relative distance $d_{f 2}$ and angle $\gamma_{f 2}$ of $F_{2}$ showed close to their references except during "wait" and "approach to TP".

\subsection{Experiment 2: line $\rightarrow$ zigzag}

Figure 18 and Figure 19 show the experimental results of a shape transition from line to zigzag. The start and goal positions of $L$ were the same as those in experiment 1. $F_{2}$ changed the shape from line to zigzag at $t=23$ [s] (shape no.=71 $\rightarrow 72$ ), selected "self" (mode flag=19) for 1 [s], then returned to N-mode (red diamond), and reached the goal in the form of zigzag. $F_{1}$, on the other hand, completed the formation control with $\mathrm{N}$-mode at all times.

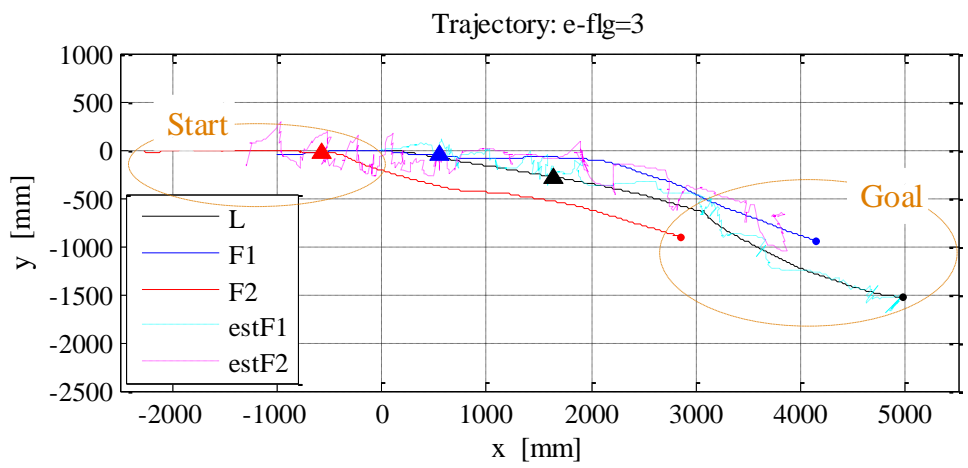

Figure 18. Traces of $L, F_{1}$, and $F_{2}$, and traces of leader position estimated by $F_{1}$ and $F_{2}$, line $\rightarrow$ zigzag 

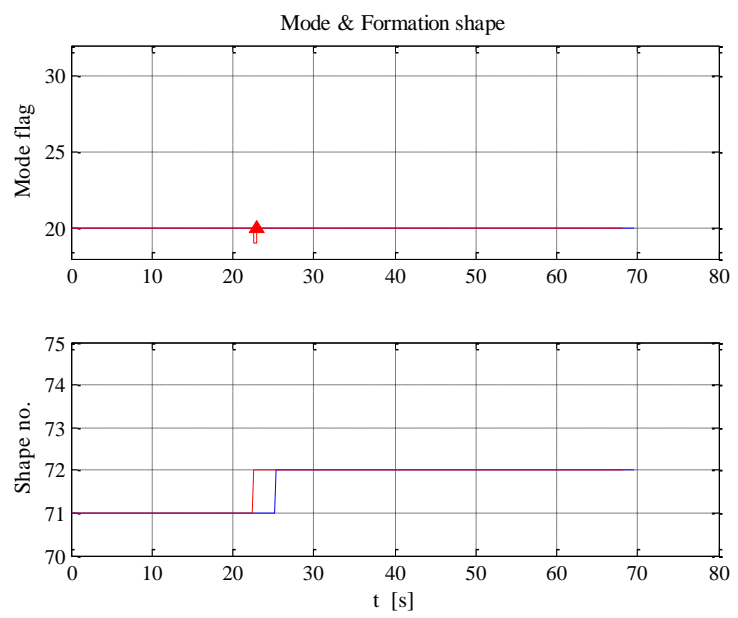

Figure 19. Time histories of mode flag and shape number, Line $\rightarrow$ Zigzag

\subsection{Experiment 3: zigzag $\rightarrow$ triangle}

Figure 20 and Figure 21 show the experimental results of a shape transition from zigzag to the triangle. The start and goal positions of $L$ were the same as those in experiment $1 . F_{2}$ changed the shape from zigzag to line with $t=23$ [s] (shape no.=72 $\rightarrow 71$ ) and returned to N-mode with "self" (mode flag=19) selected for $1\left[\mathrm{~s}\right.$ ] (red diamond). $F_{2}$ furthermore changed the shape from line to triangle at $t=42$ [s] (shape no. $=71 \rightarrow 73$ ) and then reached the goal by taking the same transitional behavior as in experiment $1 . F_{1}$, on the other hand, completed the formation control with $\mathrm{N}$-mode at all times.

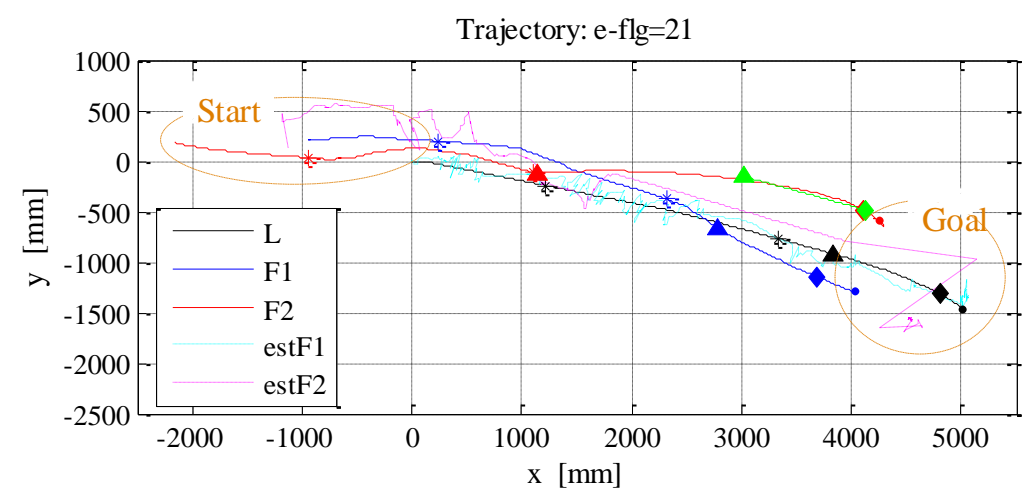

Figure 20. Traces of $L, F_{1}$, and $F_{2}$, and traces of leader position estimated by $F_{1}$ and $F_{2}$, zigzag $\rightarrow$ triangle
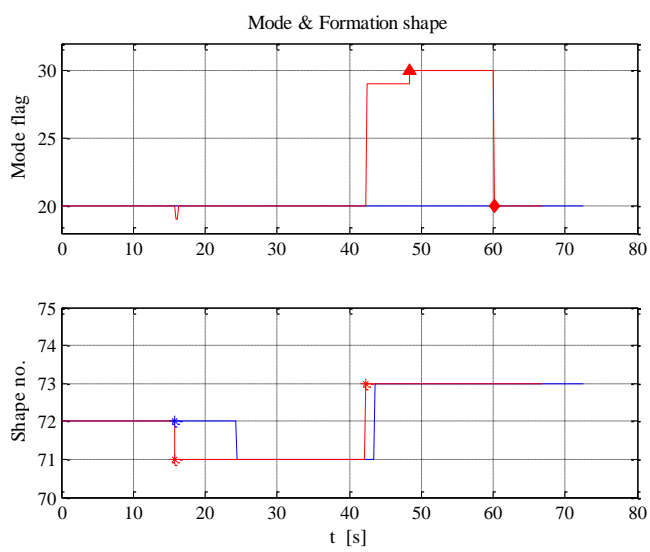

Figure 21. Time histories of mode flag and shape number, zigzag $\rightarrow$ triangle 


\section{CONCLUSION}

This paper has presented a formation shape transition technique of multiple mobile robots in the leader-follower method as a new function that gives flexibility to the formation control of mobile robots. First, we proposed basic shape transition methods for the case of two mobile robots under formation control by the leader-follower method and then extended the methods to the shape transition of three mobile robots. The effectiveness of the proposed technique was demonstrated by experiments using real mobile robots which were equipped with multiple sonar sensors.

Although the number of robots treated in this paper was two and three, the methods proposed in this paper applied to the shape transition in the number of more. In addition, in the shape transition with three mobile robots, the priority in the follower robots for performing transition movement was not changed. If it is possible to change the priority, a more efficient shape transition seems to be possible. This also leads to a more flexible shape transition with the change of the leader robot. These are future research subjects.

\section{REFERENCES}

[1] S. Tadokoro, "High-speed search robots in confined space," The Robotic Society of Japan, vol. 27, pp. 1107-1110, 2009, doi: 10.7210/jrsj.27.1107.

[2] T. Mano and S. Hamada, "Development of robotic system for nuclear facility emergency preparedness," The Robotic Society of Japan, vol. 19, pp. 714-721, 2001.

[3] R. L. Raffard, C. J. Tomlin, and S. P. Boyd, "Distributed optimization for cooperative agents: Application to formation fight," 2004 43rd IEEE Conference on Decision and Control (CDC) (IEEE Cat. No.04CH37601), 2004, pp. 2453-2459 Vol.3, doi: 10.1109/CDC.2004.1428778.

[4] S. D. Amico and O. Montenbruck, "Proximity Operations of formation-fying spacecraft using an eccentricity / inclination vector separation," Journal of Guidance, Control, and Dynamics, vol. 29, no. 3, pp. 554-563, 2006, doi: $10.2514 / 1.15114$

[5] T. Balch and R. C. Arkin, "Behavior-based formation control for multi-robotic teams," in IEEE Transactions on Robotics and Automation, vol. 14, no. 6, pp. 926-939, Dec. 1998, doi: 10.1109/70.736776.

[6] S. Monteiro and E. Bicho, "A dynamical systems approach to behavior-based formation control," Proceedings 2002 IEEE International Conference on Robotics and Automation (Cat. No.02CH37292), 2002, pp. 2606-2611 vol.3, doi: 10.1109/ROBOT.2002.1013624.

[7] J. R. T. Lawton, R. W. Beard and Brett J. Young, "A decentralized approach to formation maneuvers," in IEEE Transactions on Robotics and Automation, vol. 19, no. 6, pp. 933-941, Dec. 2003, doi: 10.1109/TRA.2003.819598.

[8] M. M. Korjani, A. Afshar, M. Norouzitallab, and M. B. Menhaj, "Dynamic autonomous agent positioning based on computational intelligence," Proceedings of the International MultiConference of Engineers and Computer Scientists 2009, 2009.

[9] W. Ren, K. L. Moore and Y. Chen, "High-order and model reference consensus algorithms in cooperative control of multivehicle systems," Journal of Dynamic Systems, Measurement, and Control, vol. 129, no. 5, pp. 678-688, 2007, doi: 10.1115/1.2764508.

[10] T. Matsuzawa, T. Hayakawa and S. Hara, "Formation control of multi-agent systems with sampled information," Proceedings of the 45th IEEE Conference on Decision and Control, 2006, pp. 4333-4338, doi: 10.1109/CDC.2006.377708.

[11] C. A. Rabbath, C. Y. Su and A. Tsourdos, "Guest Editorial Introduction to the Special Issue on Multivehicle Systems Cooperative Control With Application," in IEEE Transactions on Control Systems Technology, vol. 15, no. 4, pp. 599-600, July 2007, doi: 10.1109/TCST.2007.899150.

[12] K.-H. Tan and A. M. Lewis, "Virtual structures for high precision cooperative mobile robot control," Proceedings of IEEE/RSJ International Conference on Intelligent Robots and Systems. IROS '96, 1996, pp. 132-139 vol.1, doi: 10.1109/IROS.1996.570643.

[13] R. Fierro, P. Song, A. Das and V. Kumar, "Cooperative control of robot formations," in Cooperative Control and Optimization, vol. 66, 2002, doi: 10.1007/0-306-47536-7_5.

[14] J. P. Desai, J. P. Ostrowski and Vijay Kumar, "Modeling and control of formations of nonholonomic mobile robots," in IEEE Transactions on Robotics and Automation, vol. 17, no. 6, pp. 905-908, Dec. 2001, doi: $10.1109 / 70.976023$.

[15] A. K. Das, R. Fierro, V. Kumar, J. P. Ostrowski, J. Spletzer and C. J. Taylor, "A vision-based formation control framework," in IEEE Transactions on Robotics and Automation, vol. 18, no. 5, pp. 813-825, Oct. 2002, doi: 10.1109/TRA.2002.803463.

[16] L. Consolini, Fabio Morbidi, D. Prattichizzo and M. Tosques, "Leader-follower formation control of nonholonomic mmobile robots with input constrains," Automatica, vol. 44, no. 5, pp. 1343-1349, May 2008, doi: 10.1016/j.automatica.2007.09.019.

[17] H. G. Tanner, G. J. Pappas and V. Kumar, "Leader-to-formation stability," in IEEE Transactions on Robotics and Automation, vol. 20, no. 3, pp. 443-455, June 2004, doi: 10.1109/TRA.2004.825275.

[18] B. S. Park, J. B. Park and Y. H. Choi, "Adaptive formation control of electrically driven nonholonomic mobile robots with limited information," in IEEE Transactions on Systems, Man, and Cybernetics, Part B (Cybernetics), vol. 41, no. 4, pp. 1061-1075, Aug. 2011, doi: 10.1109/TSMCB.2011.2105475. 
[19] A. Fujimori, T. Fujimoto and G. Bohács, "Formated navigation of mobile robots using distributed leader-follower control,” IFAC Proceedings Volumes, vol. 38, no. 1, pp. 571-576, 2005, doi: 10.3182/20050703-6-CZ-1902.01365.

[20] A. Fujimori, T. Fujimoto and G. Bohács, "Mobile robot formation control using a modifed leader-follower technique," Integrated Computer-Aided Engineering, vol. 15, no. 1, pp. 71-84, Jan. 2008, doi: 10.3233/ICA-200815106.

[21] A. Fujimori, T. Saito and G. Bohács, "Formated navigation of mobile robots with obstacle avoidance," 2006 IEEE Conference on Robotics, Automation and Mechatronics, 2006, pp. 1-6, doi: 10.1109/RAMECH.2006.252622.

[22] A. Fujimori, H. Kubota, N. Shibata and Y. Tezuka, "Leader-follower formation control with obstacle avoidance using sonar-equipped mobile robots," Journal of Systems and Control Engineering, vol. 228, no. 5, pp. 303-315, 2014, doi: 10.1177/0959651813517682.

[23] A. Fujimori, A. Hosono, K. Takahashi and S. Oh-hara, "Formation control of multiple mobile robots with large obstacle avoidance," 2018 15th International Conference on Control, Automation, Robotics and Vision (ICARCV), 2018, pp. 1374-1379, doi: 10.1109/ICARCV.2018.8581326.

[24] A. Fujimori, A. Hosono, S. Kuratani and S. Oh-hara, "Formation control of multi-sonar equipped mobile robots and large obstacle avoidance," International Journal of Engineering Research and Applications, vol. 10, no. 7, pp. 4859, 2020, doi: 10.9790/9622-1007054859.

[25] N. Hovakimyan, E. Lavretsky and C. Cao, "Dynamic inversion for multivariable non-affine-in-control systems via time-scale separation," International Journal of Control, vol. 81, no. 12, pp. 1960-1967, 2008, doi: 10.1080/00207170801961295.

[26] ActivMedia Inc., Pioneer 1 Operations Manual, 1996.

[27] ActivMedia Inc., Pioneer 1 Software Manual, version 4.1.2, 1996.

[28] ActivMedia Inc., Pioneer 3 Operations Manual, version 2, 2015.

\section{BIOGRAPHIES OF AUTHORS}
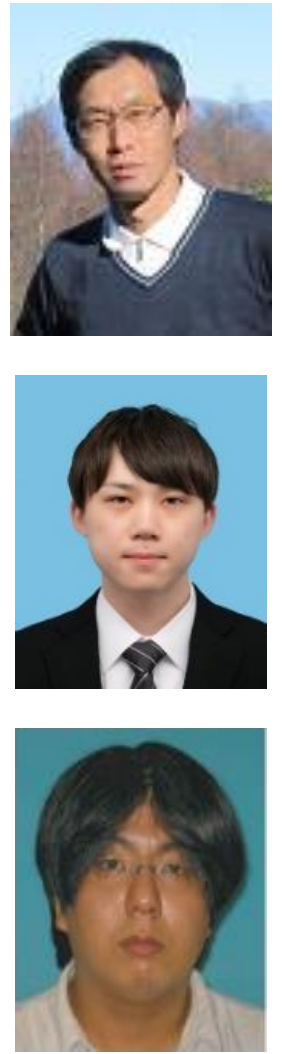

Atsushi Fujimori received the Ph.D. degree in aeronautics from Nagoya University (Japan) in 1989. Since 1990, He had worked at Shizuoka University as an Associate Professor. Since 2006, He have been working at University of Yamanashi as a Professor. His current research interests are in fields of system identification, flight control and mobile robots.

Kotaro Oh-kiri revised the Master degree in mechanical engineering from University of Yamanashi (Japan) in 2021. Since April 2021, he has been working at Terumo Cooperation.

Shinsuke Oh-hara revised the Ph.D. degree in mechanical engineering from Osaka University (Japan) in 2006. Since 2009, he has been working at University of Yamanashi as a Research Associate. His research interests include visual feedback control and mobile robot control. 